\title{
PQLI Control Strategy Model and Concepts
}

\author{
Bruce Davis • Line Lundsberg • Graham Cook
}

Published online: 17 May 2008

(C) International Society for Pharmaceutical Engineering 2008

\begin{abstract}
This paper describes an approach and technical process for developing and implementing a Control Strategy, which is a planned set of controls, derived from current product and process understanding that assures process performance and product quality. Development of a Control Strategy requires a structured process, involving a multidisciplinary team of experts, linking pharmaceutical development to the manufacturing process, and engineering controls of process equipment. The PQLI Control Strategy Team has proposed a Control Strategy Model that facilitates understanding and that may be used a cross-functional communication tool. This paper concentrates on the techniques and principles involved in developing the early Control Strategy rather than the operational implementation of the strategy.
\end{abstract}

Keywords PQLI - Design space · Criticality .

Control strategy Critical quality attribute .

Tom Garcia - Pfizer, Stephen Tyler - Abbott, Eric Ahuja - Merck, Metter Bryder - Lundbeck, Michael Hahn - Lundbeck, Ray Bolton AstraZeneca, Gordon Muirhead - GSK, Sue Busse - Lilly, Hedinn Valthorsson - Novartis

\section{B. Davis}

AstraZeneca,

Alderly House, Macclesfield,

Cheshire SK10 4TF, UK

e-mail: bruce.davis@astrazeneca.com

L. Lundsberg

NNE Pharmaplan,

29 Pine Walk,

Cobham KT11 2HJ, UK

e-mail: 1lun@nnepharmaplan.com

G. Cook $(\square)$

Wyeth Pharmaceuticals,

New Lane,

Havant PO9 2NG, UK

e-mail: cook1@wyeth.com
Critical process parameter $\cdot$ Knowledge space $\cdot \mathrm{PAT}$.

Pharmaceutical target product profile $\cdot$ Product and process knowledge $\cdot$ Quality by design - Quality risk management . API $\cdot$ Drug substance $\cdot$ Drug product

\section{Introduction}

The Product Quality Lifecycle Implementation (PQLI) initiative [7] was launched by ISPE to help industry identify global solutions to the challenges in implementing ICH Q8, Q9 and Q10 guidance [3, 5, 6], and to provide the technical framework for the implementation of key elements of Quality by Design (QbD) [1, 2, 8, 9, 11, 14, 15].

$\mathrm{QbD}$ is a systematic approach to product and process design and development. One of the key elements is design and implementation of a Control Strategy.

Control Strategy is defined in the ICH Q10 (Step 2) [6] document as:

"A planned set of controls, derived from current product and process understanding that assures process performance and product quality. The controls can include parameters and attributes related to drug substance and drug product materials and components, facility and equipment operating conditions, in-process controls, finished product specifications, and the associated methods and frequency of monitoring and control."

This Control Strategy for a particular product should be established within the framework of the overall Pharmaceutical Quality System (PQS). The Control Strategy is a comprehensive plan for ensuring that the final product meets critical requirements, and therefore the needs of the patient.

Elements of a Control Strategy can include (but are not limited to) the following: 
- Control of input material attributes (e.g., drug substance, excipients, primary packaging materials) based on an understanding of their impact on process-ability or product quality

- Product specifications

- Procedural controls

- Facility controls, such as utilities, environmental systems and operating conditions

- Controls for unit operations that have an impact on downstream processing or end-product quality (e.g. the impact of drying on degradation, particle size distribution of the granulate on dissolution)

- A monitoring program (e.g., full product testing at regular intervals) for verifying multivariate prediction models

The Control Strategy should establish the necessary controls - based on patient requirements - to be applied throughout the whole product lifecycle from product and process design through to final product, including API and Drug Product manufacture, packaging and distribution.

This paper describes an approach and technical process for developing and implementing a Control Strategy.

This paper concentrates on developing the early Control Strategy and the principles behind this. Full details of what is required at every stage of the lifecycle have not been included, but could be developed from these principles.

This should be regarded as work in progress and comment and input from industry, regulators, and suppliers is welcomed.

\section{Minimal and Enhanced Approaches}

As in ICH Q8(R) (Step 2) [4], a distinction may be drawn between a minimal and an enhanced control strategy approach.

In a Minimal Control Strategy, drug product quality is controlled primarily by intermediate and end product testing.

With an Enhanced Control Strategy drug product quality ensured by risk-based control strategy for well understood product and process, and quality controls are shifted upstream, with the possibility of real-time release or reduced end-product testing. A Design Space is established, and the Control Strategy will ensure that the product is manufactured within the Design Space.

Other aspects may include:

- Product specifications are part of the overall quality control strategy, and based on desired product performance with relevant supportive data

- Appropriate feed forward and feedback controls to provide a more reproducible product

- Process operations tracked and trended to support continual improvement efforts
Whether a minimal or enhanced approach is applied, the Control Strategy must be made operational, for example, by incorporating certain elements in the batch record and enabling execution of the Control Strategy to be linked to the decision to release the product. This paper does not cover these operational aspects.

\section{Developing the Control Strategy}

Development of a Control Strategy requires a structured process, involving a multi-disciplinary team of experts, linking pharmaceutical development to the manufacturing process, and engineering controls of process equipment.

The PQLI Control Strategy Team has proposed a Control Strategy Model that facilitates understanding and that may be used a cross-functional communication tool. This is described in detail later in this paper.

Personnel at all levels should be able to understand the way control strategy links from CQAs to operational aspects to ensure, for example that:

- Chemists understand in-process controls are established to keep the process inside the design space and seek opportunities for simplification of controls, as knowledge is gained.

- Engineers know how equipment operating conditions impact product quality.

- Quality Assurance professionals know where the highest risks are in the process.

Although the primary driver for development of a control strategy will be assurance of product safety, efficacy and quality, the Control Strategy may also ensure the meeting of other business objectives such as operator health and safety, protection of the environment, manufacturability, supply related issues, efficiency, and profitability.

Development of a Control Strategy for a product will therefore be a structured and iterative activity involving a multi-disciplinary team of experts. This team may include representatives from formulation development, drug substance development, process development, analytical development, QC, QA, Regulatory Affairs, manufacturing, engineering, and specialists in Process Analytical Technology (PAT) and chemo-metrics.

Existing risk assessment tools such as HACCP (Hazard Analysis and Critical Control Points), 'Worst Outcomes Analysis' FMECA (Failure Mode Effects and Criticality Analysis) [10] can provide a framework for Quality Risk Management.

A Control Strategy and a product release strategy are not the same, but demonstration of adherence to the Control Strategy would support the product or batch release strategy. 


\section{Control of Input Material Attributes}

Variability in the manufacturing processes may be caused by variability in the drug substance and raw materials and their attributes, when linked to a CQA.

The impact of not only chemical but also physical material attributes and their variability need to be understood. For example, for an oral solid dosage product, impact of factors such as participle size distribution, particle shape distribution, density, surface area, surface energy, flow, cohesiveness, friction, elastic modulus, amorphous content, compactibility, hygroscopicity, solubility, and static charge should be assessed.

A linkage between the product CQAs and the input material attributes should enable identification and understanding of the most critical material attributes and their impact on the product CQAs.

Controlling the variability of input materials can be managed in different ways, e.g. by functional specifications (not necessary in concurrence with compendia specifications) or by managing the variability directly in the process using closed loop controls.

One example is raw materials affected by seasonal variations in the moisture level and used in a moisture critical blend. By applying PAT tools such as NIR (Near Infrared) spectroscopy, drying can be monitored on-line and the drying process controlled to the end-point with a closed feed-backward control loop in place (see $[12,13])$. In many cases the variability in a material input can be managed by operating the process conditions differently within the Design Space.

Other input materials such as packaging material should be studied during development to identify and understand which material attributes impact the manufacturing process and final product CQAs.

\section{In-Process Controls}

In-process controls include all controls that need to be performed during processing, including control of Critical Process Parameters, in-process material attributes and components, as well as equipment and facility parameters that must be monitored or controlled to achieve the product CQAs.

Controlling the Critical Process Parameters during processing is important as they have a direct impact on the CQAs, but other parameters (that may be part of a unit operation), that have an impact on downstream processing or other end-product quality attributes not already covered by a CQA, should be monitored or controlled as well. Which parameters to monitor or control is the outcome of Quality Risk Management (QRM) activities aimed at mitigating the risks arising during manufacturing.

In-process controls could include conventional sampling and at-line analysis or on-line or in-line univariate sensors or multivariate probes (typical spectroscopy). They may be manual or automated, depending on the nature of the process itself, what needs to be measured and controlled, how often, scale, process time, and the nature of the manufacturing equipment.

\section{Verifying Multivariate Prediction Models}

Implementing PAT for in-process and or release controls relies heavily on the application of multivariate prediction models, also known as process models. These models can either predict CQAs or CPPs or a combination of both. The nature of these models requires frequent updates. How frequently depends on the maturity of the model (e.g. the amount of data and their variability within the model), as well as the kind of data that has been included to reflect variability in scale, equipment, analytical set-up, sampling, or site.

A monitoring program for verifying the models should be established and be based on a risk assessment of the model itself and include possible ways to verify the model by other means e.g. by comparing the predicted CQA value to a conventional analytical method. Verifying predicted CPPs can sometimes be difficult because, for example, sampling is not possible or there are no conventional laboratory methods. In these cases other ways of verification should be proposed. The monitoring program should include requirements for when a model has to be updated (e.g. latest batch information, change of raw material supplier, increased knowledge and experience over time).

\section{Control Strategy Model}

The ISPE PQLI Control Strategy Team has proposed a Control Strategy Model that facilitates understanding and that may be used a cross-functional communication tool (see Fig. 1).

The model seeks to link the attributes of the product that are important to the patient, to the controls in the manufacturing process that are needed to deliver those attributes, and also to show in parallel the business requirements.

The starting point is normally the Critical Quality Attributes (i.e evolved from the desired Pharmaceutical Target Product Profile) and the end point is the distribution of manufactured product itself. The Control Strategy should link from pharmaceutical drug development through manufacturing including process engineering equipment control.

This model shows:

- Three levels showing links from the finished product CQAs and other objectives through the manufacturing operations to the controls by which these are achieved.

- Two columns distinguishing between patient and business requirements. 
Fig. 1 The PQLI control strategy model

\section{PQLI Control Strategy Model}

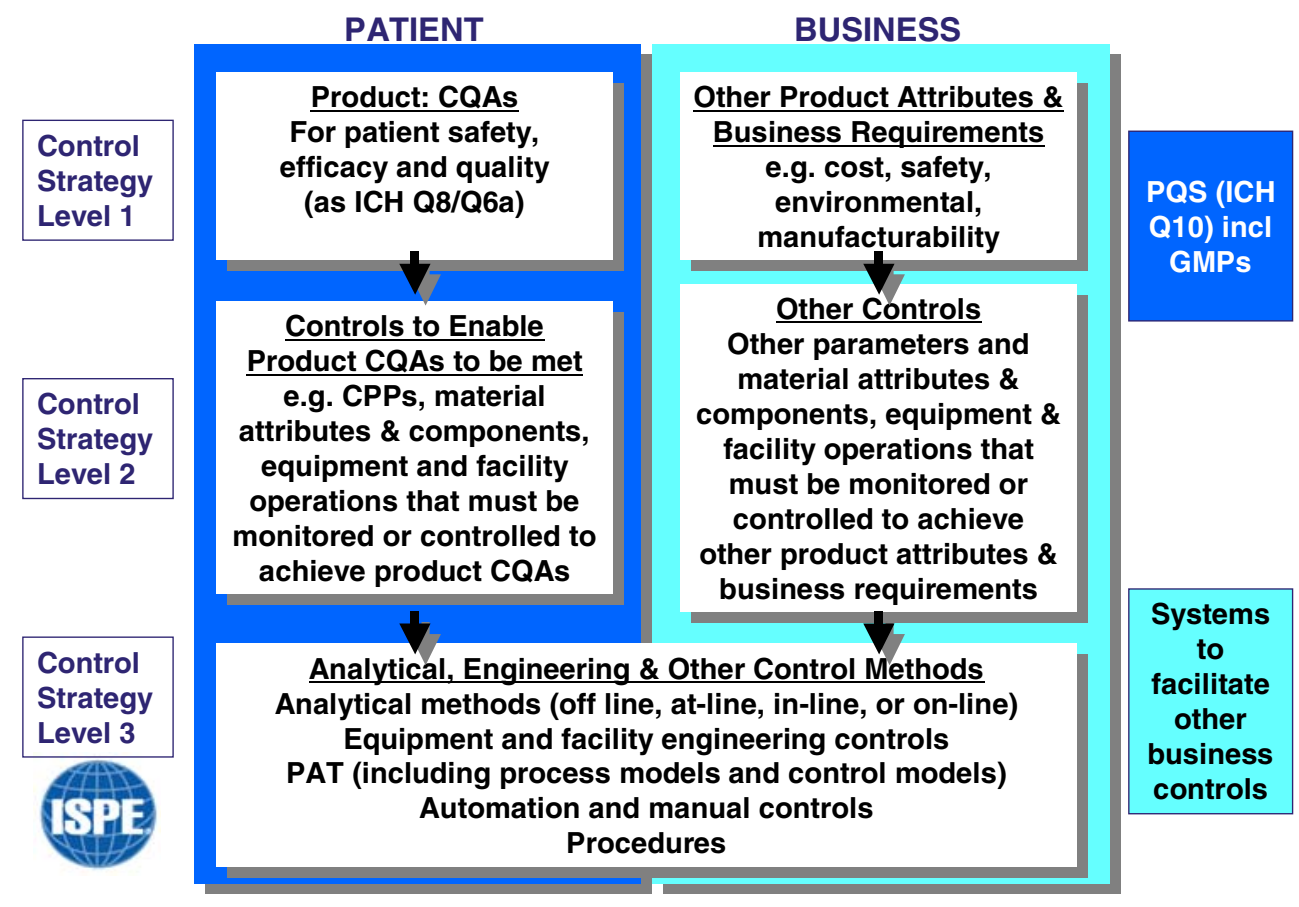

At Level 1 the Critical Quality Attributes (CQAs) and other requirements are identified.

Level 2 considers the critical process parameters, material attributes and components involved in meeting the CQA requirements.

Level 3 covers the actual analytical, automation, and other controls of the Level 2 identified parameters and attributes.

Note that product specific patient-related Quality Attributes need to work within a Pharmaceutical Quality System (PQS), as per ICH Q10 (shown by the dark blue background to the Patient side) whereas business-related product aspects may be governed by other requirements, (shown by the light blue background to the Business side).

The exact boundary between the PQS and the systems to facilitate other business controls (dark and light blue boundary) is linked to the overall design of a company's work processes.

The term Control Strategy can mean different things to different functions. Engineers often use the term Control Strategy to mean how plant and equipment is controlled to enable manufacture, and although this may be true at plant level, it is important that the plant level requirements have a logical link back to both patient and business product requirements, and support the Pharmaceutical Quality System and business systems.

The model highlights areas of importance, i.e. related to patient safety, efficacy and quality and serves as a framework for understanding the future controls that need to be established and how they will impact the batch release strategy. The Model logic may facilitate regulatory discussion.

\section{Application of the Model}

At Control Strategy Level 1 the Critical Quality Attributes (CQAs) of the product are identified. Those product Quality Attributes that directly relate to patient safety, efficacy and quality are derived from the Pharmaceutical Target Product Profile using prior knowledge, clinical experience and product and process understanding.

Business requirements that are not directly related to patient safety, efficacy and quality but are important to the business are also identified. These may include cost, efficiency, operator safety, environmental protection, manufacturability, other customer needs, and supply related objectives. This concept of 'manufacturability' relates to the attributes of a product that are not directly related to patient safety, efficacy or quality (included in a specification approved as part of a regulatory submission), but which may be important for customer satisfaction, such as minimizing cosmetic defects.

For an oral solid product, Drug Substance attributes considered may include appearance, identity, polymorphism, crystal habit, assay, surface energy, impurities, solvents, water content, particle size, sulphonated ash, and metals. For Drug Product, attributes such as friability, identification, appearance, dissolution, assay, content uniformity, degradation, purity, microbiology, and packaging are normally considered.

Decisions on what is critical or non-critical are taken, and may be based on criteria and concepts discussed in the related paper PQLI Criticality [16] in this Volume of the 
Journal of Pharmaceutical Innovation. As process understanding and product and process experience grow, this knowledge can be applied to continuously improve the process (ICH Q10). An attribute that was thought to be non-critical may turn out later to be critical, or vice versa. Companies will typically determine what is critical or otherwise based on a risk assessment and then develop a Control Strategy from this basis to ensure the product remains within the Design Space. Note that a Control Strategy or test does not make a CQA or CPP non-critical; but rather makes it controlled.

Some companies may choose several levels of criticality to describe multiple levels of risk, e.g. the use of the terms "key" or "important" as well as "critical" and "noncritical". For example, as process parameter boundaries approach edges of the proven acceptable range (PAR) or failure point, if known, the level of criticality normally increases with the level of risk. Companies may decide to define an additional category to address those parameters that are lower risk than for critical process parameters e.g., a product parameter is well controlled in relation to the required boundaries and is of lower risk, and therefore would only be described in the Pharmaceutical Quality System.

Control Strategy Level 2 considers the means by which the CQAs and other business requirements determined at Control Strategy Level 1, will be assured e.g. what attributes of the starting materials, reagents, solvents, process aids etc. must be monitored or controlled, and what parameters and operating conditions of the process equipment and manufacturing facility need to be monitored or controlled to ensure the objectives defined at Control Strategy Level 1 are achieved.

CQAs can be measured and evaluated directly or, alternatively, indirectly for raw material or other material attributes identified to impact the CQAs. The control of all CPPs and those process parameters contained in the intermediate category (if applicable), should ensure the CQAS are being delivered and hence the process is maintained within the Design Space

At Control Strategy Level 3 the analytical and other controls methods are described. The information will include the measurement technologies for the material attributes or equipment parameters (off-line, at-line, in-line or on-line), univariate or multivariate process models and control models, as well as procedural and engineering controls of the plant including automation systems, closed control loops, normal operating ranges, and alarms.

Note that controlling plant and equipment covers both sides of the model and, practically, cannot be separated (e.g. a PLC, a plant controller or a data capture system will be set up to control all parameters). There will not be two separate engineering control systems, such as one for patient-related product requirements and one for businessrelated requirements.

Level 3 should also provide information about the intended multivariate process models to be used, including what these process models will do, and how they will be operated and maintained. The process model should be maintained within the company's own Pharmaceutical Quality System.

The Control Strategy is implemented within a framework comprising the Pharmaceutical Quality System and Good Manufacturing Practices for the patient-related product attributes, and other aspects such as Environmental, Health and Safety systems, and financial systems for the business-related requirements. Certain aspects of the business-related requirements and controls may need to be described in the Control Strategy for the patient-related aspects to facilitate an understanding of the logic and rationale for the Control Strategy as a whole.

\section{Developing and Implementing a Control Strategy}

Once the product CQAs have been identified at Control Strategy Level 1, the Control Strategy Level 2 can be developed, by addressing questions such as:

- What needs to be controlled?

- Where in the process does control need to be implemented?

- Which quality attributes of the starting materials, intermediate materials, reagents, components, and process parameters and operating conditions of the process equipment and facility systems must be controlled to assure a final product CQA?

For each finished product CQA the factors to be considered include:

- Physical, chemical and biological attributes of the starting materials, reagents, components and in-process material used to manufacture the drug substance and drug product

- Equipment operation and design, parameters and operating conditions in the manufacturing process for the drug substance and drug product

- Facility utilities, environmental systems and operating conditions

- Specifications or acceptance criteria for the attributes and parameters considered above

- Facility and process standard operating procedures

- Risks associated with failure of an element of the control system

At Control Strategy Level 2 a number of different approaches may be adopted to control a CQA - hence 
leading to the selection of one particular Control Strategy over another.

Control Strategy Level 3 addresses questions such as:

- How are these critical process parameters and material attributes controlled so that product quality is assured?

- What happens if a control fails?

- Can failure be detected?

- How can the risk to product quality be mitigated?

- Can a back-up or secondary control be used?

- How is the control implemented?'

Typical controls include:

- Off-line testing of an attribute of a starting material, component or in-process material

- Monitoring and feedback control of a material attribute during processing (manual or automated)

- Monitoring and feedback control of an equipment parameter during processing (manual or automated)

- Monitoring and feed-forward control of a material attribute or process parameter (manual or automated)

- Procedural control of an operation

- Equipment and facility design

- Process design, for example the addition of specific operations during drug substance manufacture to reduce the level of impurities

Details to consider when implementing a control include:

- Methods and frequency of monitoring and control

- Sampling, including sample size and sampling methodology

- Calibration of analysers and sensors

- Procedures to be followed in the event of a malfunction

Risk Assessment during the Development of a Control Strategy

A risk assessment of the manufacturing process may need to consider a number of factors, including reliability of the control system and malfunction procedures, or the need for redundancy, such as replication of sensors and other elements of the system to ensure control in the event of a failure.

Risk mitigation for a critical measurement may include an additional sensor or an alternate means of confirming conformance to the acceptance criteria e.g. through use of first principles or off-line testing to demonstrate a state of control. If a process model is critical to assuring the operation remains within the Design Space then the failure of an analyser or sensor (e.g. sending incorrect data to the model), the control (e.g. responding incorrectly to an output from the model) or the model itself to work as intended should be considered.

An example of how the proposed model can be used with the Hazard Analysis and Critical Control Points (HACCP) seven step process is given below.

- Step 1 in the HACCP process defines Control Strategy Level 1 (definition of the CQAs of the finished product)

- Steps 2-4 define Control Strategy Level 2 (the "what' and 'where')

- Steps 5-6 define Control Strategy Level 3 (the 'how'):

1. Define the product and process

- Understand the product (dosage form, pack, storage, therapeutic use, patient population etc.)

- Define Finished Product CQAs

- Prepare a process flow map

2. Identify the potential hazards and potential control measures

- What factors affect the finished product CQAs? (starting materials, equipment, personnel, environment etc.)

- What are the Critical Process Parameters?

- What are the starting material and in process material CQAs?

3. Determine the Critical Control Points

- Where must control be implemented?

4. Establish the Critical limits for each Critical Control Point

- May be derived from Design Space

- May determine the nature of technology used for monitoring and control

5. Establish a monitoring system for each Critical Control Point

- e.g. timely data for feedback control of process

6. Establish Corrective Action plans to re-establish control when necessary

- Use of 'secondary controls'

7. Establish Verification procedures to demonstrate compliance

- Qualification of controls

- Trending of data

- Periodic quality review

\section{Control Strategy Verification}

Once a Control Strategy has been developed it should be implemented and verified under the company's Quality Management System. A robust change control system 
should be in place to document changes, assess the impact and ensure that changes do not adversely affect the Control Strategy and thereby the intermediate or final product quality.

Verification of the Control Strategy will involve an assessment that the controls will ensure the product CQAs. This may include verification of the individual controls and continuous quality verification of the manufacturing process.

When implementing PAT, verifying predicted CPPs can be difficult, because sampling is not possible, for example, because there are no conventional laboratory methods. In these cases alternate approaches to verification should be proposed.

\section{Control Strategy and the Product Lifecycle}

The Control Strategy is related to the level of process understanding at a given time, and evolves as manufacturing experience increases. The originally specified measures, controls or models may be modified or even removed, or the need for additional controls may be identified. Other revisions to the Control Strategy may relate to continual improvement, for example the introduction of improved analyser or control technology.

Periodic reviews of risk assessments and mitigation should be conducted to determine the appropriateness of the Control Strategy based on product manufacturing history. Failure or deviations should be investigated and the effectiveness of the control system considered in relation to the identified root cause. Corrective and preventive actions should be applied and the Control Strategy updated as necessary (including any regulatory actions required) in the light of new product and process knowledge.

Implementing PAT in the Control Strategy will require the application of process models (multivariate prediction models) that either predicts CQAs or CPPs or a combination of both. These models may require frequent updates, depending on the maturity of the model (e.g., the amount of data and their variability within the model), as well as the kind of data that has been included to reflect variability in scale, equipment, analytical set-up, sampling, and site. A monitoring program for verifying the validity of process models should be established and be based on a risk analysis of the model itself and include possible ways to verify the model by other means. One example would be to compare the predicted CQA value to a conventional analytical method. The monitoring program should include requirements for when a model has to be updated (e.g. change of raw material supplier or deviations resulting in increased knowledge).

\section{Case Study Example 1}

The following is an example from manufacturing a drug substance. In this case, impurity A has been identified as critical, whereas impurity B has no impact on patient safety and efficacy.

\section{Control Strategy Level 1}

Impurity A is identified as a CQA. There is a business requirement that solvent emissions are limited to comply with environmental legislation and minimise costs.

\section{Control Strategy Level 2}

During development it has been identified that three different parameters have a significant impact on the level of impurity A being generated. They are:

- $\mathrm{CPP}_{1}=$ Temperature

- $\mathrm{CPP}_{2}=\mathrm{pH}$

- $\mathrm{CPP}_{3}=$ Reaction End-point

\section{Control Strategy Level 3}

These parameters were studied extensively by Design of Experiment (DOE) to ensure the Design Space boundary is known. Measurement technologies proposed for these parameters are:

- $\mathrm{CPP}_{1}$ : On-line temperature sensor

- $\mathrm{CPP}_{2}$ : On-line $\mathrm{pH}$ sensor

- $\mathrm{CPP}_{3}$ : Reaction end-point determination

The process model is a multivariate model incorporating the measurements from the three different technologies. Solvent limits are specified, and an end of line solvent recovery plant is installed, to meet local authority limits, and business cost drivers.

Near Infrared (NIR) measurement technology is applied to control the reaction end-point.

The controls to ensure the CQA (impurity A) and solvent emissions are integrated into a control model, taking information from the NIR, temperature and $\mathrm{pH}$ sensors, as well as monitoring solvent emission level. Information at this level will also include the frequency of testing, monitoring, sampling, and calibration.

\section{Overall Presentation of the Control Strategy}

The objective and requirements for control of the CQA should be presented. The relevant control points for reaction end-point, temperature and $\mathrm{pH}$ should be presented 
demonstrating that impurity A will meet the specification in the finished product.

Details of the process and control model are given to demonstrate how the process parameters are controlled. This will cover how the input from the $\mathrm{pH}$ sensor, temperature sensor and the NIR are combined in a model and the output used to control the temperature of the reactor $\mathrm{pH}$ adjustment and reaction end-point, and so predict the level of impurity A.

\section{Case Study Example 2}

For simplicity and clarity only two quality attributes of the product will be considered, one patient-related, the other business-related. (Reference should be made to Fig. 2.).

\section{Control Strategy Level 1}

In this hypothetical example the product is a small molecule drug presented as an injection dosage form - a liquid in a vial - where the drug substance is susceptible to metalcatalysed oxidative degradation, enhanced by the presence of certain process impurities. The level of these oxidative degradants in the dosage form is a CQA. There is a business requirement that the cost of the overall process is minimized.

\section{Control Strategy Level 2}

The process is reviewed to determine which material and component attributes and process parameters must be controlled. For the oxidative degradants there may be a number of points in the process at which control might be required, including:

- metal content of the starting materials for the synthesis of the drug substance

- metal content of the solvents and reagents used in the synthesis of the drug substance

- reaction conditions to minimise formation or ensure removal of process impurities

- use of non-metallic product contact materials in the process

- metal content of the excipients used in the drug product

Drug Substance Manufacture:
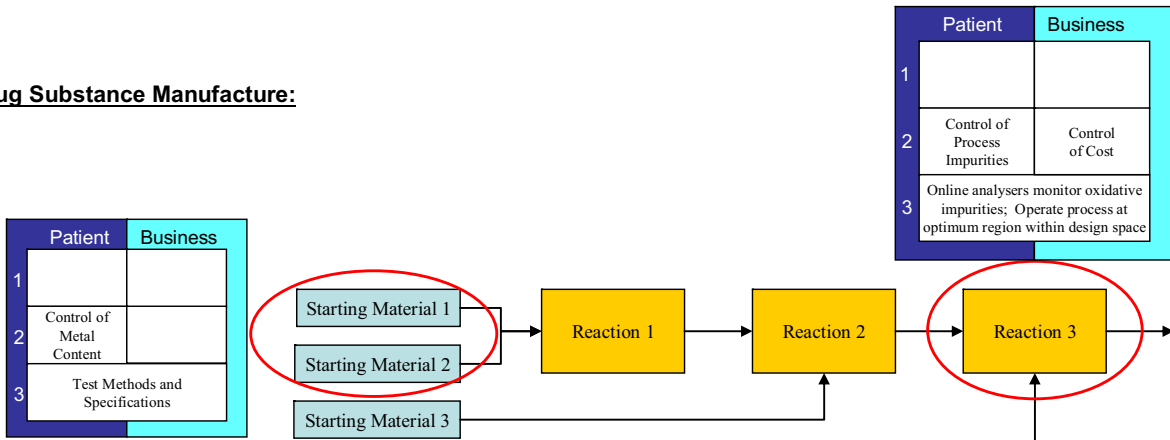

Starting Material 4

Drug Product Manufacture:
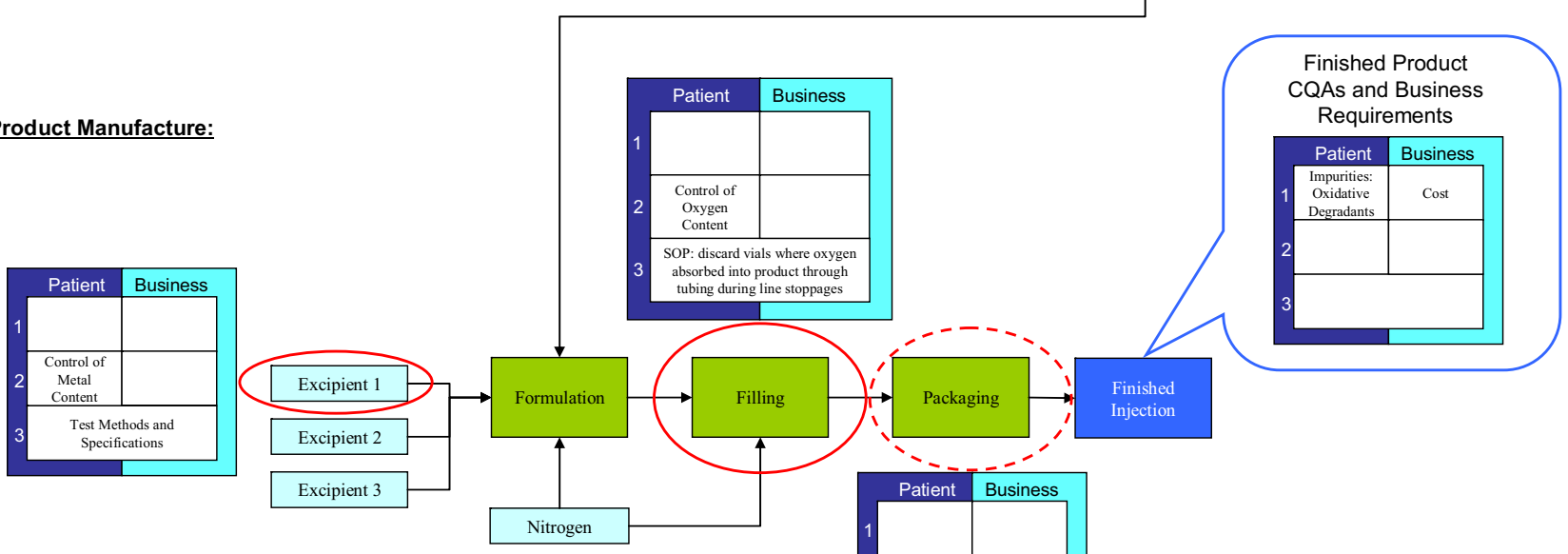

Fig. 2 Control strategy example 
- absorption of oxygen by the liquid product held in filling lines etc.

- oxygen content of headspace in vials

- container-closure integrity to avoid oxygen ingress

In this example, based on the product and process knowledge gained during development of the product, the company has determined that control of several of these factors is necessary to assure that the level of oxidative degradants in the finished product is below the maximum allowable levels.

A combination of process design and procedural controls is employed to ensure the use of non-metallic contact materials, such as glass-lined process vessels. Low oxygen permeable tubing is used for the manufacturing process.

To control the metal content of the starting materials and reagents the company decided to apply specifications and test the materials identified through risk assessment as providing the greatest risk to the product - starting materials 1 and 2 and excipient 1 . The company considered the specifications for the starting materials and decided to apply tight specifications for certain attributes of starting materials 1 and 2 to avoid the need for an additional downstream processing step to reduce process impurities.

This, however, has limited the choice of suppliers of these starting materials who can meet the specifications. An alternative strategy, in which wider specifications for these materials would be applied and a downstream purification step introduced, was rejected following a risk assessment that concluded that the potential to form a genotoxic impurity was greater and risk mitigation would be considerably more expensive.

Product development knowledge and prior knowledge had shown that control of reaction 2 was not critical to the formation of the relevant process impurities for this CQA. Although the company considered using traditional timebased end-points to control reaction 3 for the drug substance manufacture it decided to develop a Design Space and adopt a Control Strategy in which the reaction conditions are adjusted to keep process impurities below specified limits. This is achieved through the use of online sensors and process analysers to monitor the process conditions and formation of intermediates, feeding the data into a process model to enable feedback control of reaction temperatures and the addition of starting material 4 , and to ensure that the process operates within the design space.

This strategy had the advantage that the business requirement to minimize the costs could be achieved by adapting the process model to target operation within a region of the Design Space that minimizes costs, while ensuring the process impurities meet specifications. In the drug product manufacturing process a further procedural control is used to ensure certain vials are discarded after filling line stoppages, and nitrogen sparging and purging is used to minimize the oxygen content of the liquid and vial headspace.

\section{Control Strategy Level 3}

The practical implementation of these controls includes:

- The testing methods for the metal content of starting materials, reagents and excipients

- The online sensors and analysers to monitor reaction conditions and their associated process models and control systems

- The SOP for actions to be taken after stoppages of the filling line

The company identified Fourier Transform Infrared (FTIR) spectroscopy as an appropriate analyzer technology to monitor reaction 3 . The proposed definition for a Control Strategy from ICH Q10 also includes consideration of the '...frequency of monitoring and control'. The company determined that collecting FTIR spectra every 5 minutes during the 7 hours reaction 3 is run was sufficient to monitor the progress of the reaction and adjust process conditions as necessary.

The consequences of failures were also considered:

- Can the failure be detected?

- Can the effect of the failure be reversed?

- What redundancy is required to reduce risks to tolerable levels?

Risk assessment concluded that the nitrogen purge is a critical processing step and failure would be difficult to detect. The company therefore decided to mitigate this risk by online monitoring of the headspace for each vial so that unacceptable vials are identified and rejected. A further risk assessment considered the impact of the failure of this additional control and identified ways of mitigating this risk.

The selection and combination of these controls, applied throughout the manufacturing process ensures that the oxidative degradants will be at an acceptable level in the finished injection. The Control Strategy is illustrated in Fig. 2.

\section{References}

1. Product Quality Lifecycle Implementation: Practical Approach to QbD, ISPE Washington Conference, Arlington, VA, June 6-7, 2007.

2. Product Quality Lifecycle Implementation (PQLI): Practical Approach to Quality by Design, ISPE 2007 Berlin Conference, Berlin, Germany, 19-September-2007.

3. International Conference on Harmonisation of Technical Requirements for Registration of Pharmaceuticals for Human Use, ICH 
Harmonised Tripartite Guideline, Pharmaceutical Development Q8, Step 4, 10-November-2005.

4. International Conference on Harmonisation, ICH Draft: Step 1, Q8 (R1) Pharmaceutical Development Revision 1, Step 3, November 2007.

5. International Conference on Harmonisation of Technical Requirements for Registration of Pharmaceuticals for Human Use, ICH Harmonised Tripartite Guideline, Quality Risk Management Q9, Step 4, 9-November-2005.

6. International Conference on Harmonisation of Technical Requirements for Registration of Pharmaceuticals for Human Use, ICH Harmonised Tripartite Guideline, Pharmaceutical Quality System Q10, Step 2, 9-May-2007.

7. Draft PQLI Summary Update Report, Version V04, Product Quality Lifecycle Implementation (PQLI), ISPE, 14-September2007, http://www.ispe.org/cs/pqli_product_quality_lifeycle_ implementation_/draft_pqli_summary_update_report.

8. E-RC3a - Product Quality Lifecycle Implementation (PQLI): A Practical Approach to QbD, 2007 ISPE Annual Meeting, 5November-2007, Las Vegas, Nevada.

9. E-RC3b-PQLI Design Qualification and Design Review (Continued), 2007 ISPE Annual Meeting, 6-November-2007, Las Vegas, Nevada.

10. Procedures for Performing a Failure Mode, Effects and Criticality Analysis, United States Military Procedure MIL-P-1629, 9November-1949.
11. Pharmaceutical CGMPs for the 21st Century: A Risk-Based Approach; A Science and Risk-Based Approach to Product Quality Regulation Incorporating an Integrated Quality Systems Approach, United States Department of Health and Human Services, U.S. Food and Drug Administration, 21-August-2002 (http://www.fda.gov/oc/guidance/gmp.html).

12. PAT - A Framework for Innovative Pharmaceutical Development, Manufacturing, and Quality Assurance, United States Department of Health and Human Services, U.S. Food and Drug Administration, Center for Drug Evaluation and Research (CDER), Center for Veterinary Medicine (CVM), Office of Regulatory Affairs (ORA), Pharmaceutical CGMPs, September 2004.

13. EMEA PAT Team Position Paper, http://www.emea.europa.eu/ Inspections/docs/PATGuidance.pdf

14. ISPE Copenhagen PQLI Conference 9-11th April 2008

15. PQLI Key Topics - Criticality, Design Space and Control Strategy DOI 10.1007/s12247-008-9032-4

16. PQLI Criticality DOI 10.1007/s12247-008-9033-3

If you would like to provide comments to the authors regarding this paper, email feedback@ispe.org and include "PQLI Control Strategy" in the subject line. 